\title{
Flow of a Fluid with Pressure-Dependent Viscosity through Variable Permeability Porous Layer
}

\author{
M.S. ABU ZAYTOON \\ Department of Mathematics \\ University of Petra, Amman \\ JORDAN \\ YIYUN (LISA) XIAO \\ Department of Mathematics and Statistics \\ University of New Brunswick, P.O. Box 5050, Saint John, N.B. \\ CANADA \\ M.H. HAMDAN* \\ Department of Mathematics and Statistics \\ University of New Brunswick, P.O. Box 5050, Saint John, N.B. \\ CANADA
}

\begin{abstract}
In this work, we consider flow of a fluid with pressure-dependent viscosity down an inclined porous plane with variable permeability that is incorporated in the pressure-dependent drag coefficient. We provide a solution to a recently developed flow model, and study the effects of flow and domain parameters (viscosity control parameter, permeability proportionality constant, and angle of inclination) on the flow characteristics. Suitability of a variable permeability model that considers permeability proportional to the flow velocity is investigated. Results show that large values of the permeability proportionality constant have little or no effects on flow characteristics.
\end{abstract}

Key-Words: - Pressure-dependent Viscosity; Inclined Porous Layer; Variable Permeability

Received: May 20, 2021. Revised: September 21, 2021. Accepted: October 7, 2021. Published: October 25, 2021.

\section{Introduction}

Flow through porous media has a host of well established applications that include the study of groundwater movement, oil and gas recovery, flow through membranes and kidney dialysis, modelling of heat and mass transfer in porous configurations, irrigation problems, and the drying of solids (see [1$3]$ and the references therein). Mathematical models of flow through porous media are classified as Darcian and non-Darcian models, and have been extensively reviewed by many authors (cf. [1]).
In recent years, however, there has been an increasing interest in flow through porous media of fluids with pressure-dependent viscosities. This interest might be attributed to emerging applications that require modelling of flows of special fluids in porous media, such as carbon sequestration in enhanced oil recovery, lubrication theory with high pressures, filtration problems, and in the pharmaceutical industry, among others, (see [5-8] and the references therein).

Although interest in pressure-dependent viscosity fluids dates back to the nineteenth century, [9], and the works of Stokes [10] and Barus [11], [12], models 
of flow through porous media of fluids with pressuredependent viscosities have only been developed over the past two decades.

The pioneering work of Rajagopal [13] and coworkers (cf. [12-21]) includes all generalized models of flow through porous media (Darcy's, Forchheimer's and Brinkman's) that they derived using mixture theory, homogenization, and thermodynamic balance. The generalized Brinkman equation was considered by Kannan and Rajagopal, [17]. who carried out extensive analysis on the forms of viscosity and Darcy drag as functions of pressure using the physical configuration of flow down an inclined porous channel.

In the current work, we employ the configuration of flow down an inclined porous channel in the analysis of a model recently developed based on intrinsic volume averaging procedure, [2]. In order to offer a comparison between the developed model and the Navier-Stokes equations, we provide the following overview.

The steady flow of an incompressible fluid with variable viscosity is governed by two conservation principles, namely conservation of mass and momentum (Nevier-Stokes), given by, [7]:

$$
\nabla \cdot \vec{v}=0
$$

$\rho(\vec{v} \cdot \nabla) \vec{v}=-\nabla p+\nabla \cdot 2 \mu \mathbf{D}(\vec{v})+\rho \vec{g}$

$\mathbf{D}(\overrightarrow{\mathrm{v}})=\frac{1}{2}\left(\nabla \overrightarrow{\mathrm{v}}+(\nabla \overrightarrow{\mathrm{v}})^{\mathrm{T}}\right)$

where $\vec{v}$ is the velocity vector field, $\rho$ is the fluid density, $p$ is the pressure, $\mu$ is the fluid viscosity, $\vec{g}$ is the gravitational acceleration, $\nabla$ is the gradient operator and $\nabla^{2}$ is the Laplacian.

The Navier-Stokes equation are partial differential equations which describe the microscopic flow in free space. The term $\mu \nabla^{2} \vec{v}$ is the viscous shear, and $(\vec{v} \cdot \nabla) \vec{v}$ is the convective acceleration. Equations (1) and (2) represent an under-determined system of four scalar equations in the five unknowns $\vec{v}, p$ and $\mu$.

In the absence of additional conservation principles to provide an additional equation, it has long been recognized that viscosity can be expressed as a function of pressure to provide an additional condition to render the governing system of equations determinate. Barus, $[11,12]$ provided the following relation between viscosity and pressure: $\mu=\mu_{0} e^{\alpha\left(p-p_{0}\right)}$

where $\mu$ is fluid viscosity, $\mu_{0}$ is a reference viscosity, $p$ is pressure and $\alpha>0$ is a constant.

Equations (1), (2) and (3) now represent a determinate system of five scalar equations in five unknowns, and governed the flow of what is termed a fluid with pressure-dependent viscosity.

When flow is taken through a porous medium, Navier-Stokes equations are valid microscopically in the pore space. However, they are hard to track due to complexity of the pore space. Furthermore, changes in flow quantities at the microscopic scale are at times insignificant at the macroscopic scale. This motivated work in modelling the macroscopic behavior of fluid quantities by averaging them over the pore space and the solid volume under the assumption that the quantities are valid everywhere in the medium.

In flow of fluids with pressure-dependent viscosities through isotropic porous media, Abu Zaytoon et.al, [2] applied the method of intrinsic volume averaging to equations (1) and (2), and arrived at the following system of equations:

$$
\begin{aligned}
& \nabla \cdot \vec{u}=0 \\
& \rho(\vec{u} \cdot \nabla) \vec{u}=-\nabla \bar{p}+\nabla \cdot 2 \mu \mathbf{D}(\overrightarrow{\mathrm{u}})-\lambda(\bar{p}) \vec{u}+\rho \vec{G} \\
& \mathbf{D}(\overrightarrow{\mathrm{u}})=\frac{1}{2}\left(\nabla \overrightarrow{\mathrm{u}}+(\nabla \overrightarrow{\mathrm{u}})^{\mathrm{T}}\right) \\
& \left.\bar{\mu}=\mu_{0} e^{\alpha\left(\bar{p}-p_{0}\right.}\right)
\end{aligned}
$$

where $\bar{\mu}$ is the average viscosity as a function of the average pressure $\bar{p}, \rho \vec{G}$ is the average body force, $\vec{u}$ is the average velocity. The functions $\bar{\mu}(\bar{p})$ and $\lambda(\bar{p})$ control variations in viscosity due to pressure, and variations in pressure due to variations in porous parameters. We suggest here the following approximation to $\lambda(\bar{p})$, in which $k(\vec{x})$ is the variable permeability of the porous medium:

$\lambda(\bar{p})=\frac{\bar{\mu}}{k(\vec{x})}$

Clearly, this model explicitly takes into account permeability of the porous medium into its Darcy drag term, whether the permeability is constant or variable. It is worth noting that momentum equation (5) reduces to Brinkman's generalized equation when convective acceleration terms are ignored. 
For Brinkman's equation, Hamdan and Kamel [3] derived a smooth variable permeability function for flow through a channel bounded by two impermeable plates, with a permeability that falls to zero on the solid walls, and reaches its maximum at the centre of the channel. Clearly, this permeability function is quadratic in the lateral variable, much like the flow velocity.

In the current work, we consider variations in permeability to be proportional to the velocity of the flow. This results in a scaling of the Darcy drag function with respect to flow velocity. We provide solution to the resulting governing equation and study the effects of flow and medium parameters on the flow characteristics.

\section{Problem Formulation}

Consider the flow of a fluid with pressure-dependent viscosity through a porous sediment of depth $h$ inclined at angle $\vartheta$ to the horizontal. The flow configuration is illustrated in Fig. $\mathbf{1}$ and shows the orientation of the coordinate system used. It is assumed that the porous sediment is bounded by impermeable, solid walls on which the no-slip condition is applied. This configuration has been used in the study of entry conditions to channels, [22], and might be used in the study of coastal groundwater modelling, [23].

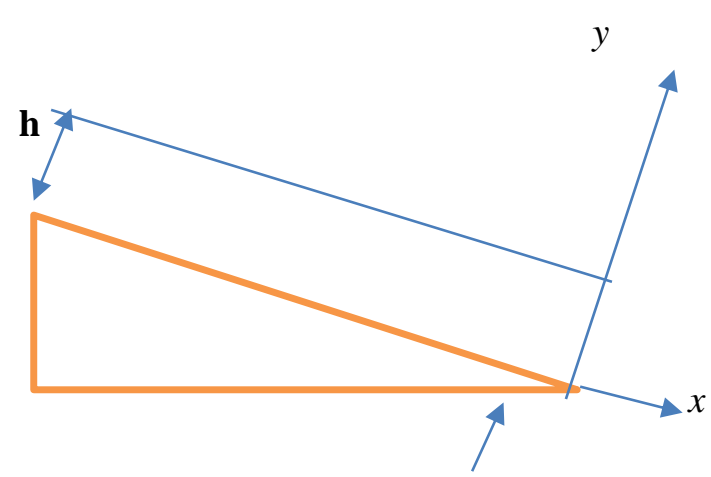

Angle $\vartheta$

Fig. 1. Representative sketch

Flow in the above domain is governed by the equation of continuity (4) and momentum equations (5), which reduce to the following set of equations when the flow is through the configuration of Fig. 1: $-\frac{d p}{d x}+\mu \frac{d^{2} u}{d y^{2}}+\frac{d \mu}{d y} \frac{d u}{d y}+\rho g \sin \vartheta-\frac{\mu}{k} u=0$

$-\frac{d p}{d y}-\rho g \cos \vartheta=0$

with boundary conditions given by zero-slip on the solid boundaries $\mathrm{y}=0$ and $\mathrm{y}=\mathrm{h}$, and a prescribed pressure at $\mathrm{y}=\mathrm{h}$ (such as atmospheric pressure, say $\left.p_{0}\right)$. Boundary conditions are thus given as:

$u(0)=0, u(h)=U, \quad p(h)=p_{0}$

\section{Problem Solution}

Following Kanaan and Rajagopal, [17], we assume that $p=p(y)$ and introduce the dimensionless quantities with respect to channel width $h$ and characteristic velocity $U$ :

$y^{*}=\frac{y}{h} ; \quad u^{*}=\frac{u}{U} ; \quad k^{*}=\frac{k}{h^{2}}$

Dropping the asterisks “*”, then boundary conditions (11) take the form

$u(0)=0, \quad u(1)=1, \quad p(1)=p_{0}$

and governing equations (8) and (9) can be written, respectively, as

$\mu \frac{d^{2} u}{d y^{2}}+\frac{d \mu}{d y} \frac{d u}{d y}+\frac{\rho g h^{2}}{U} \sin \vartheta-\frac{\mu}{k} u=0$

$\frac{d p}{d y}=-\rho g h \cos \vartheta$

General solution to (14) takes the form

$p=-\rho g h \cos \vartheta y+c$

where $c$ is an arbitrary constant.

Using pressure condition $p(1)=p_{0}$ we find that

$c=p_{0}+\rho g h \cos \vartheta$

and (15) takes the form

$p=p_{0}+(1-y) \rho g h \cos \vartheta=\left[p_{0}+\rho g h \cos \vartheta\right]-$

$\rho g h \cos v y$ 
In order to solve (13) for $u(y)$, we assume that the viscosity varies with pressure according to:

$\left.\mu(p)=\mu_{0} e^{\alpha\left(p-p_{0}\right.}\right)$

From (3) and (17), we obtain

$\frac{d \mu}{d y}=-\rho g h \cos \vartheta \alpha \mu_{0} e^{\alpha\left(p-p_{0}\right)}$

Using (19) in (13), we obtain

$\frac{d^{2} u}{d y^{2}}-\alpha \rho g h \cos \vartheta \frac{d u}{d y}-\frac{u}{k}=-\frac{\rho g h^{2} \sin \vartheta}{U \mu_{0} e^{\alpha\left(p-p_{0}\right)}}$

Following Hamdan and Kamel [3] we assume that the variable permeability in the flow through a channel is proportional to velocity, then we can write $k(y)=k_{0} u$, where $k_{0}$ is a reference constant permeability. Equation (19) can be written in the form

$\frac{d^{2} u}{d y^{2}}-A_{1} \frac{d u}{d y}=\frac{1}{k_{0}}-A_{3} e^{(y-1) A_{1}}$

where

$A_{1}=\alpha \rho g h \cos \vartheta$

$A_{3}=\frac{\rho g h^{2} \sin \vartheta}{U \mu_{0}}$

Solution to equation (21) subject to no-slip conditions (12) is given by:

$u(y)=-\frac{y}{A_{1} k_{0}}-A_{3} e^{-A_{1}}\left[\frac{y e^{A_{1} y}}{A_{1}}-\frac{e^{A_{1} y}}{\left(A_{1}\right)^{2}}\right]+\frac{C_{1} e^{A_{1} y}}{A_{1}}+$ $C_{2}$

where

$C_{1}=\frac{A_{3}}{A_{1}} \frac{\left[e^{-A_{1}}-1\right]}{\left[e^{A_{1}-1}\right]}+\frac{1}{\left[e^{A_{1}-1}\right] k_{0}}+\frac{A_{3}}{\left[e^{A_{1}-1}\right]}$

$C_{2}=-\left[\frac{A_{3} e^{-A_{1}}}{\left(A_{1}\right)^{2}}\right]-\frac{C_{1}}{A_{1}}$

From (24), we obtain the following vorticity, $\omega(y)$, and shear stress, $\tau(y)$, respectively, across the porous layer:

$\omega=-\frac{d u}{d y}=\left[\frac{1}{A_{1} k_{0}}+A_{3} e^{-A_{1}} y e^{A_{1} y}-C_{1} e^{A_{1} y}\right]$

$$
\begin{aligned}
& \tau(y)=\mu \frac{d u}{d y}=-\mu \omega=-e^{\frac{(1-y) A_{1}}{\alpha}}\left[\frac{1}{A_{1} k_{0}}+\right. \\
& \left.A_{3} e^{-A_{1}} y e^{A_{1} y}-C_{1} e^{A_{1} y}\right]
\end{aligned}
$$

The above solution is exact and is favoured to numerical solutions, such as a finite difference solution or a boundary/finite element solution, which are possible in this case.

\section{Results and Discussion}

Results have been obtained for the following values of parameters:

$k_{0}=0.1,1$ and 100

$\alpha=0.1$ and 1

$A_{1}=1,2,5$ and 10

$A_{3}=2,3,5$ and 100 .

\subsection{Pressure and Viscosity Distributions}

Equation (17) gives the linearly decreasing variations in pressure across the channel. Maximum pressure is at the lower channel wall, $p(0)=p_{0}+\rho g h \cos \vartheta$, and minimum pressure at the upper channel wall, $p(1)=p_{0}$.

Viscosity decreases exponentially with pressure across the channel according to Barus' relation, equation (18). Viscosity reaches its minimum, $\mu\left(p_{0}\right)=\mu_{0}$, at the upper channel wall and reaches its maximum, $\mu\left(p_{0}+\rho g h \cos \vartheta\right)=\mu_{0} e^{\alpha \rho g h \cos \vartheta}$, at the lower channel wall. The Darcy drag coefficient of the form of equation (6) was used in this work.

As recognized by Kannan and Rajagopal [17], $\mathrm{A}_{1}$ is a measure of the effect of gravity versus the effect of the pressure on the viscous dissipation within the fluid, while $\mathrm{A}_{3}$ compares the relative effects of gravity and viscosity. We conclude that increasing $\mathrm{A}_{1}$ means increasing $\alpha$ for a fixed $\vartheta$, or decreasing $\vartheta$ for a fixed $\alpha$, while increasing A3 means decreasing $\mu_{0}$ for a fixed $\vartheta$, or increasing $\vartheta$ for a fixed $\mu_{0}$.

\subsection{Velocity Profiles}

The effects of increasing $A_{1}$, with all other parameters fixed, are shown in Fig. 2. $A_{1}$ is a measure 
of the effect of gravity versus the effect of the pressure on the viscous dissipation within the fluid. Increasing $A_{1}$ means increasing $\alpha$ for a fixed $\vartheta$, or decreasing $\vartheta$ for a fixed $\alpha$.

When $\alpha$ increases, fluid viscosity increases and the flow is slower. When angle of inclination $\vartheta$ decreases, effects of gravity on the flowing fluid decrease, thus resulting in a slower flow. Fig. 2 also shows that in the lower region of the channel, viscosity is higher than in upper regions, due to the exponential decrease in viscosity as we move from lower to upper channel wall. The existence of a viscosity differential results in greater loss of parabolicity of the velocity graphs with increasing $\mathrm{A}_{1}$.

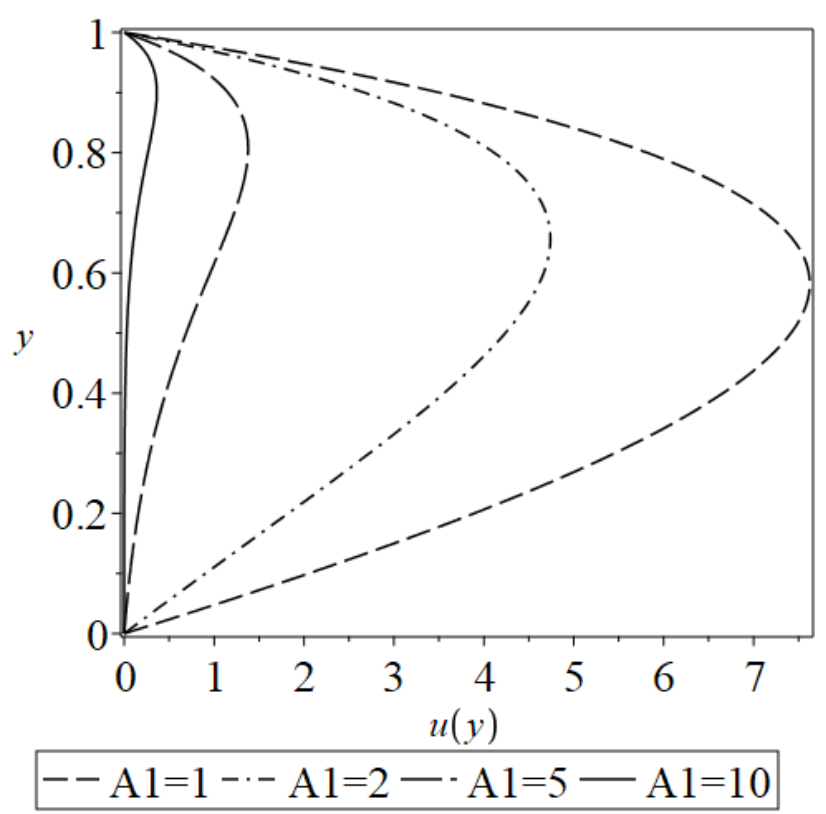

Fig. 2 Velocity Profiles for Various $A_{1}$.

$$
A_{3}=100 ; k_{0}=10
$$

Effects of increasing $A_{3}$, with all other parameters fixed, are shown in Fig. 3. $A_{3}$ compares the relative effects of gravity and viscosity. Increasing A3 is associated with decreasing $\mu_{0}$ for a fixed $\vartheta$, or increasing $\vartheta$ for a fixed $\mu_{0}$. Decreasing $\mu_{0}$ for a fixed $\vartheta$ results in decreasing the pressure-dependent viscosity across the channel, which has the effect of enhancing the flow (increasing velocity). Increasing $\vartheta$ for a fixed $\mu_{0}$ enhances the effect of gravity on the flow and increases velocity. Both of these cases result in increasing velocity across the channel with increasing $\mathrm{A}_{3}$, as demonstrated in Fig. 3 . We note that the velocity profiles in Fig. 3 are quadratic and close to being parabolic when $A_{3}$ inxcreases.

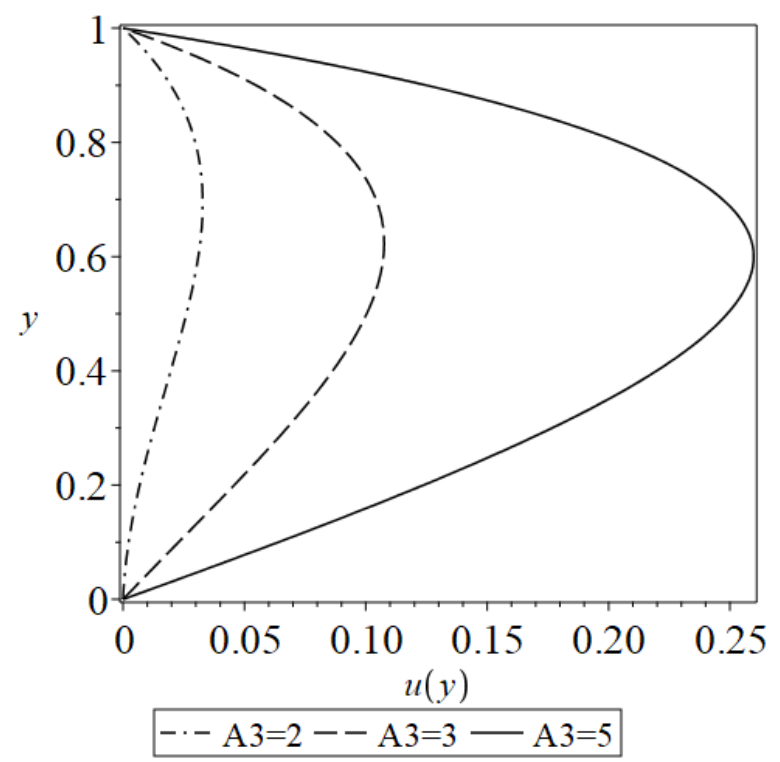

Fig. 3 Velocity Profiles for Various $A_{3}$.

$$
A_{1}=1 ; k_{0}=1
$$

Effects of the permeability function coefficient $k_{0}$ on the velocity profile across the channel is illustrated in Fig. 4, for fixed choices of $A_{1}$ and $A_{3}$. It shows that increasing $k_{0}$ results in increasing the velocity across the channel. This behaviour is expected in light of the fact that associated with increasing $k_{0}$ is higher permeability, hence larger velocity.

While this is noticeable when $k_{0}$ increases by tenfold from $k_{0}=0.1$ to $k_{0}=1$, the relative increase in velocity is less significant as $k_{0}$ increases by a hundred-fold from $k_{0}=1$ to $k_{0}=100$. This might be attributed the fact that we tight in the variable permeability to the variable velocity by making them proportional to each other. The net effect is the scaling of the viscosity function, making it less significant in the Darcy drag coefficient and replacing it with a constant $1 / k_{0}$ in the forcing function of equation (21) and its solution, equation (24). The term $\frac{y}{A_{1} k_{0}}$ in solution (24), where $k_{0}$ appears, loses its contribution to the velocity profile with increasing $k_{0}$. 


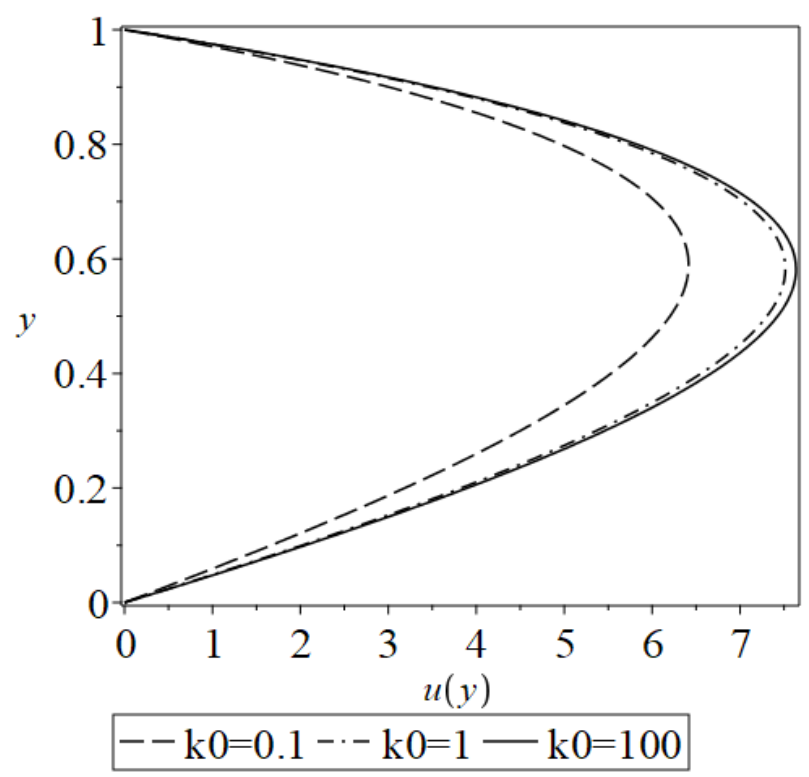

Fig. 4 Velocity Profiles for Various $k_{0}$. $A_{1}=1 ; A_{3}=100$

\subsection{Vorticity and Shear Stress}

The term $\frac{y}{A_{1} k_{0}}$ in solution (24), where $k_{0}$ appears, loses its contribution to the vorticity profile with increasing $k_{0}$ and influences the vorticity across the channel, as shown in Fig. 5. For $k_{0} \geq 1$, vorticity tends to become independent of $k_{0}$.

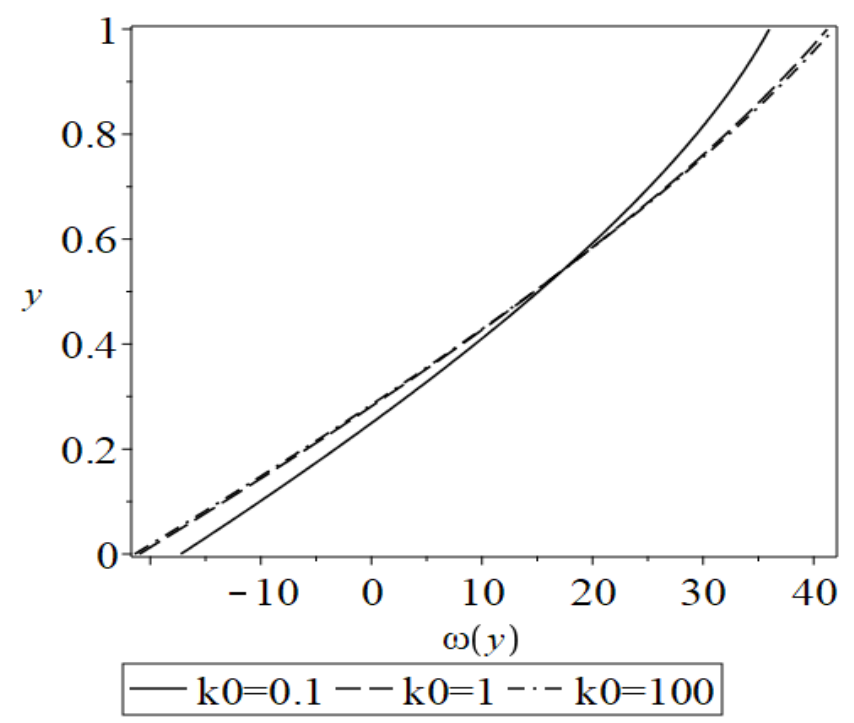

Fig. 5 Vorticity Profiles for Various $k_{0}$.

$$
A_{1}=1 ; A_{3}=100
$$

With increasing $A_{1}$, lower wall vorticity increases and upper wall vorticity decreases. Vorticity increases for most of the channel except in upper regions close to the upper wall, approximately in the upper $10 \%$ of the channel) where it starts decreasing with increasing $A_{1}$. At the lowr and upper walls, vorticities are of opposite signs for all values of $A_{1}$. With increasing $A_{3}$, lower wall vorticity decreases and upper wall vorticity increases.

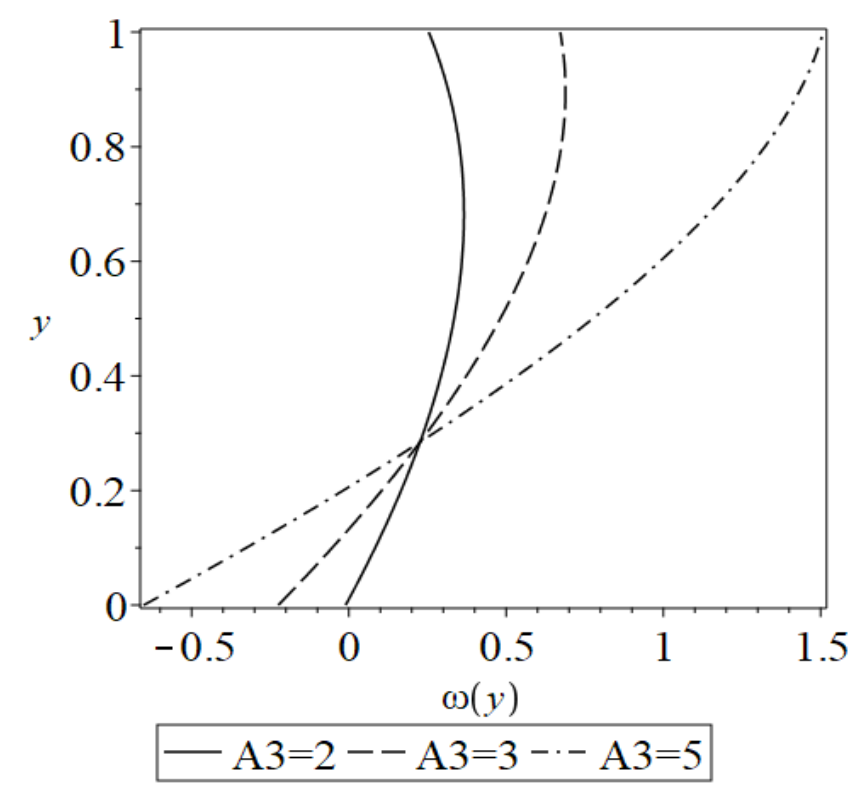

Fig. 6 Vorticity Profiles for Various $A_{3}$.

$$
A_{1}=1 ; k_{0}=1
$$

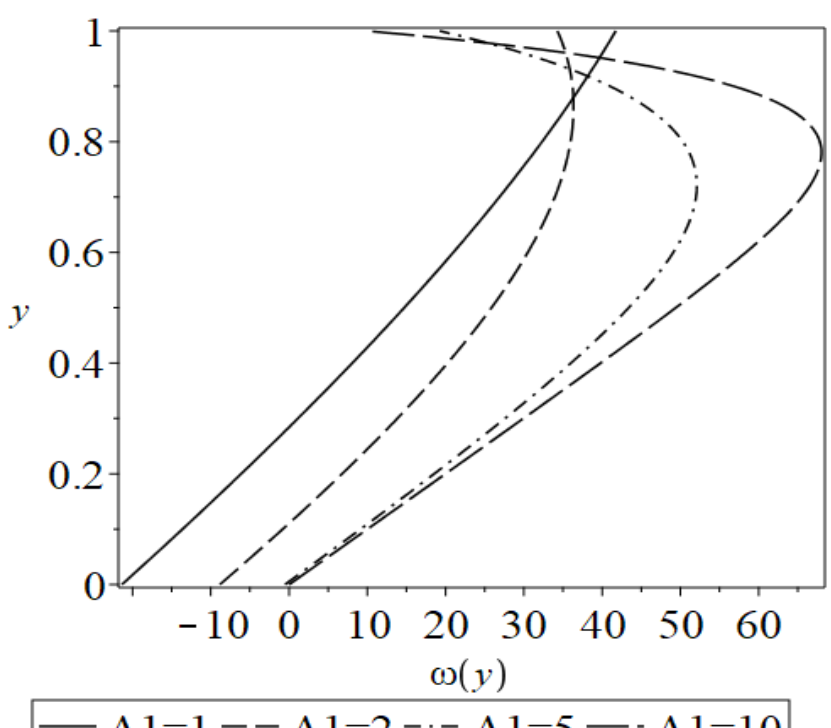

Fig. 7 Vorticity Profiles for Various $A_{1}$.

$$
A_{3}=100 ; k_{0}=10
$$


Shear stress across the channel is given by equation (28). While shear stress behaviour can be concluded from vorticity behaviour, we illustrate in Fig. 8 its dependence on $\alpha$. Fig. 8 shows the increase in shear stress for an increase from $\alpha=0.1$ to $\alpha=1$. At the lower wall, shear stress is positive, while it is negative at the upper wall. This is in line with Fig. 6, which shows wall vorticities are negative at the lower wall and positive at the upper wall. Shear stress and vorticity are of opposite signs, as per equation (28).

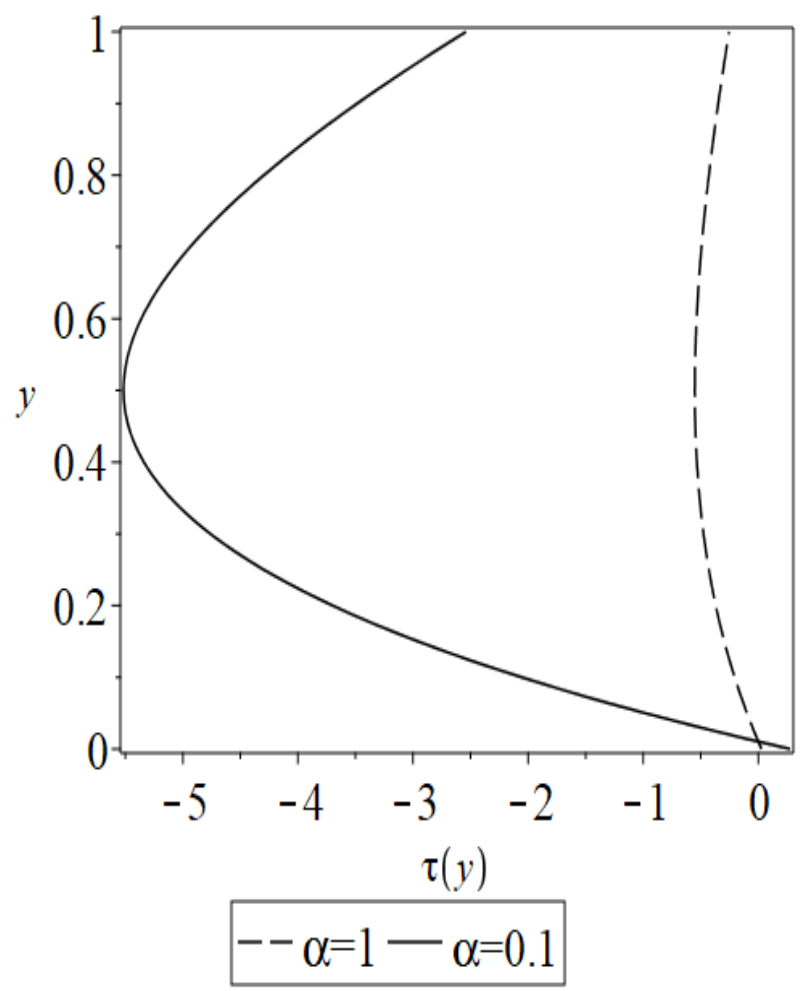

Fig. 8 Shear Stress for $\alpha=0.1$ and 1 . $A_{1}=1 ; A_{3}=2 ; k_{0}=1$

\section{Conclusion}

In this work, we considered flow of a pressuredependent viscosity fluid down an inclined porous plane with variable permeability. The goal was to provide a solution to a recently developed flow model, and to study the effects of flow and domain parameters on the flow characteristics. Suitability of a variable permeability model that considers permeability proportional to the flow velocity was examined. Results of this work support the following conclusions.

a) Parameters $A_{1}, A_{3}$ defined by equations (22) and (23), permeability coefficient $k_{0}$, used in the permeability function $k(y)=k_{0} u$, and $\alpha$, the viscosity control parameter of equation (18), are the most important parameters that control the flow patterns. Parameter $A_{1}$ increases with increasing $\alpha$.

b) Increasing $A_{1}$, while other parameters are fixed, decreases the velocity across the channel.

c) Increasing $A_{3}$, while other parameters are fixed, increases the velocity across the channel.

d) The term $\frac{y}{A_{1} k_{0}}$ in solution (24), where $k_{0}$ appears, loses its contribution to the velocity profile with increasing $k_{0}$.

e) For $k_{0} \geq 1$, velocity and vorticity across the channel lose their dependence on $k_{0}$.

f) Shear stress across the channel increases with increasing $\alpha$. 


\section{References:}

[1] Rudraiah, N., Coupled Parallel Flows in a Channel and a Bounding Porous Medium of Finite Thickness. J. Fluids Engineering, ASME, V o 1. 107, 1985, pp. 322-329.

[2] Abu Zaytoon, M.S., Allan, F.M., Alderson, T.L. and Hamdan, M.H., Averaged Equations of Flow of Fluid with Pressure-dependent Viscosity through Porous Media", Elixir Appl. Math. Vol. 96, 2016, pp. 41336-41340.

[3] Hamdan, M.H. and Kamel, M.T. (2011). Flow through Variable Permeability Porous Layers. Adv. Theor. Appl. Mech., 4(3), 135-145.

[4] Martinez-Boza, F.J., Martin-Alfonso, M.J., Callegos, C. and Fernandez, M., High-pressure Behavior of Intermediate Fuel Oils. Energy Fuels, Vol. 25, 2011, pp. 5138-5144.

[5] Fusi, L., Farina, A. and Rosso, F., Mathematical Models for Fluids with Pressure-dependent Viscosity Flowing in Porous Media, International Journal of Engineering Science, Vol. 87, 2015, pp. 110-118.

[6] Szeri, A.Z., Fluid Film Lubrication: Theory and Design, Cambridge University Press, 1998.

[7] Housiadas, K.D., Georgiou, G.C. and Tanner, R.I., A Note on the Unbounded Creeping Flow Past a Sphere for Newtonian Fluids with Pressure-dependent Viscosity, International Journal of Engineering Science, Vol. 86, 2015, pp. 1-9.

[8] Chang, J., Nakashatrala, K.B. and Reddy, J.N., Modification to Darcy-Forchheimer Model Due to Pressure-dependent Viscosity: Consequences and Numerical Solutions. J. Porous Media, Vol. 20\#3, 2017, pp. 263-285.

[9] Bridgman, P.W., The Physics of High Pressure, MacMillan, New York, 1931.

[10] Stokes, G.G., On the Theories of the Internal Friction of Fluids in Motion, and of the Equilibrium and Motion of Elastic Solids, Trans. Camb. Philos. Soc., Vol. 8, 1845, pp. 287-305.
[11] Barus, C.J., Note on Dependence of Viscosity on Pressure and Temperature, Proceedings of the American Academy, Vol. 27, 1891, pp. 13-19.

[12] Barus, C.J., Isothermals, Isopiestics and Isometrics Relative to Viscosity, American Journal of Science, Vol. 45, 1893, 87-96.

[13] Rajagopal KR. On a Hierarchy of Approximate Models for Flows of Incompressible Fluids through Porous Solids. Mathematical Models and Methods in Applied Sciences, Vol. 17, 2007, pp. 215-252.

[14] Hron, J., Malek, J. and Rajagopal, K.R., Simple Flows of Fluids with Pressure-Dependent Viscosities, Proceedings of the Royal Society, Vol. 457, 2001, pp. 1603-1622.

[15] Nakshatrala, K.B. and Rajagopal, K.R., A Numerical Study of Fluids with PressureDependent Viscosity Flowing through a Rigid Porous Medium, Int. J. Numer. Meth. Fluids, Vol. 67, 2011, pp. 342-368.

[16] Rajagopal, K.R., Saccomandi, G. and Vergori, L., Flow of Fluids with Pressure- and ShearDependent Viscosity Down an Inclined Plane, Journal of Fluid Mechanics, Vol. 706, 2012, pp. 173-189.

[17] Kannan, K. and Rajagopal, K.R., Flow through Porous Media due to High Pressure Gradients, Applied Mathematics and Computation, Vol. 199, 2008, pp. 748-759.

[18] Savatorova, V.L. and Rajagopal, K.R., Homogenization of a Generalization of Brinkman's Equation for the Flow of a Fluid with Pressure Dependent Viscosity through a Rigid Porous Solid, ZAMM, Vol. 91 No. 8, 2011, pp. 630-648.

[19] Srinivasan, S., Bonito A. and Rajagopal, K.R., Flow of a Fluid through a Porous Solid Due to High Pressure Gradient, Journal of Porous Media, Vol. 16, 2013, pp. 193-203.

[20] Srinivasan, S. and Rajagopal, K.R., A Thermodynamic Basis for the Derivation of the Darcy, Forchheimer and Brinkman Models for Flows through Porous Media and their Generalizations, International Journal of NonLinear Mechanics, Vol. 58, 2014, pp. 162-166. 
[21] Subramanian, S.C. and Rajagopal, K.R., A Note on the Flow through Porous Solids at High Pressures, Computers and Mathematics with Applications, Vol. 53, 2007, pp. 260-275.

[22] Abu Zaytoon, M.S., Hamdan, M.H. and Xiao, Y., Generalized models of flow of a fluid with pressure-dependent viscosity through porous channels: channel entry conditions. Int. J. Physical Res., Vol. 9(2), 2021, pp. 84-91.

[23] Goswami, G., Basack, S., Mastorakis, N., Saikia, A., Nilo, B. and Ahmed, N., Coastal ground water flow and management: a State-of-the-Art review, Int. journal of Mechanics, Vol. 14, 2020, pp. 37-48.

\section{Contribution of individual authors to the creation of a scientific article}

All authors contributed to literature review, problem formulation, and independently solving the governing equations.

M.S. Abu Zaytoon produced results and graphs. Y. Xiao and M.H. Hamdan analysed the results. M.H. Hamdan wrote the manuscript.

\section{Sources of funding}

There were no sources of funding for this work.

\section{Creative Commons Attribution License 4.0 (Attribution 4.0 International , CC BY 4.0)}

This article is published under the terms of the Creative Commons Attribution License 4.0 https://creativecommons.org/licenses/by/4.0/deed.en US 